\title{
A Remote Automatic Test System with High Precision for AIS Performance
}

\author{
Qing Hu, Xiao Zhang* and Shufang Zhang \\ Information Science and Technology College, Dalian Maritime University, Dalian, China \\ *Corresponding author
}

\begin{abstract}
Performance of Automatic Identification System (AIS) transmitter is very important for AIS network reliability and marine safety. In order to ensure the performance, a high precision AIS transmitter automatic test system based on Virtual Instrument Software Architecture (VISA) and the Standard Commands for Programmable Instruments (SCPI) is proposed and designed in this paper. The test system can be controlled remotely by connecting the computer with a spectrum analyzer through a local area network (LAN) interface, and it is able to accomplish the testing task of AIS transmitter performance automatically and efficiently, The testing method put forward in this paper is significantly superior to traditional methods in its accuracy of quantitative measurement, clarity of results and efficiency of analysis.
\end{abstract}

Keywords-AIS performance automatic test system; spectrum analyzer; VISA; SCPI; LAN

\section{INTRODUCTION}

AIS is a communication system for exchanging maritime information such as static information, dynamic information and voyage information, etc between ship and ship or ship and shore for ship navigation safety [1]. It was approved that AIS equipment was mandatory on the ship in 1997 by International Maritime Organization (IMO). At present, AIS has been widely applied to allover maritime field.

The product specification of AIS station is defined in the ITU-R M.1371 recommendation, which defines the performance parameters for AIS station transceiver in detail. And There are a number of types of AIS devices, and each product type has been defined by the IMO and International Electrotechnical Commission (IEC). The IMO and IEC has also published and maintained a series of formal international specifications which are called the AIS Product Standards. These standards define the exact performance and functionality requirements of each AIS device. In order to guarantee the interoperability of AIS devices within the AIS network, it is crucial that every AIS device operates in full accordance with the AIS product standards as published by the IMO and IEC.

The performance of an AIS transceiver is critical to its operation and interaction with other devices using the AIS network. In spite of the international standards published by the IEC, AIS technology is now demanding to develop and achieve full compliance with the requirements. Some AIS products which do not fully meet the required standard have the potential to interfere with the communication between other AIS devices and may cause serious safety and security risks, including: 1 . to block AIS position reports between vessels resulting a navigation or collision safety concern; 2 . to block shore based reception of AIS position reports from vessels in an area of interest resulting in a homeland security concern; 3. to interfere with the correct operation of other radio equipment operating in the marine VHF band resulting in loss of communication and a safety concern[2].

Consequently, it becomes essential to make every AIS device in the AIS network fully tested against all the requirements contained in the relevant IEC specifications to ensure ongoing integrity, reliability and safe operation of the network.

So far, this test is carried out by manual method in most cases, not only time-consuming, but lack of quantitative support. As technology has advanced, a large number of programmable scientific instruments have been put into use. At the same time, newer and higher requirements on measurement of AIS devices have been put forward, such as the increasing complexity of testing items, the soaring amount of tests, the faster testing speed and the higher accuracy of the measurement data. In view of this situation, traditional way of testing without making the most of this state-of-art technology of scientific instruments is difficult to meet the practical requirements yet. Therefore, the development of a fully functional AIS performance automatic test system instead of manual operation has become a major and inevitably trend.

Many researchers have established a variety of special automatic test systems by using the computer to control the programmable scientific instruments remotely, as reported in literature[3-8]. However, studies on AIS device automatic test system are relatively scarce.

In this paper, a high precision remote automatic test system of AIS Performance based on the international standard IEC 62320-2 (using for AIS Aids to Navigation (AtoN) stations)[9]is designed and developed to overcome the limitations and deficiencies mentioned above.

\section{ARCHITECTURE OF SYSTEM}

The architecture of the AIS performance automatic test system is illustrated as Figure 1. The AIS performance automatic test system consists of a transmitter under test, a power attenuator, a spectrum analyzer(Agilent E4000A), a computer with a LAN interface and an ATE control software. 
The transmitter under test is connected to the spectrum analyzer by using the power attenuator to meet the input power requirements of the spectrum analyzer. The computer is regarded as the hardware platform. The measured data can be acquired by an ATE control software designed in this paper, with the help of a LAN interface. The LAN interface is selected to achieve the remote control of the spectrum analyzer for several reasons: firstly, ATE systems based on LAN interface are lower cost and easier to set up; secondly, LAN interface link is more stable and efficient especially when more than two instruments are connected to the ATE system or the instruments are away from the computer; thirdly, LAN interface breaks through the limit on the distance of remote control instrument, which is the most obvious advantage of LAN interface.

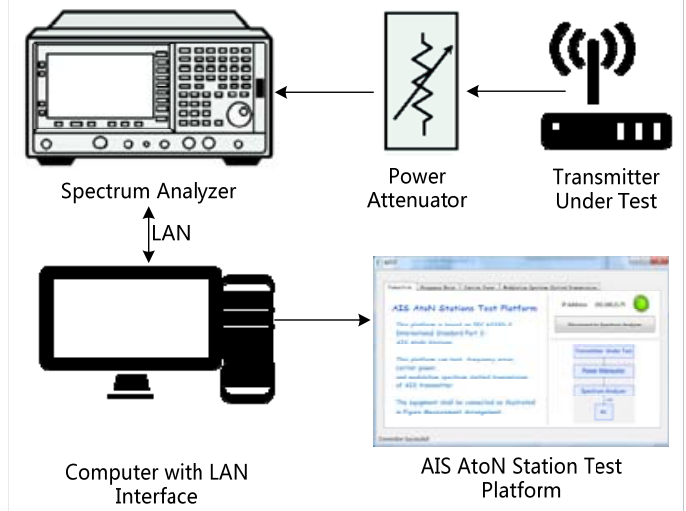

FIGURE I. THE REAL SYSTEM OF THE AIS PERFORMANCE AUTOMATIC TEST SYSTEM.

An An intelligent control ATE software called the AIS AtoN Station Test Platform is designed in the setup to implement remote automatic measurements. It is developed under Qt Creator environment, and it provides the remote control functions that fill testing needs, including automatic instrument connection and configuration, real-time data acquisition and preservation, and intuitionistic results analysis and display.

Once the video trigger level of spectrum analyzer is set properly, measurements of the attenuated test signals will be carried out according to test requirements automatically. The data obtained by the spectrum analyzer are read and stored in the computer for post processing. This will be discussed in more detail in the next section.

The picture of real system is shown in Figure 2.

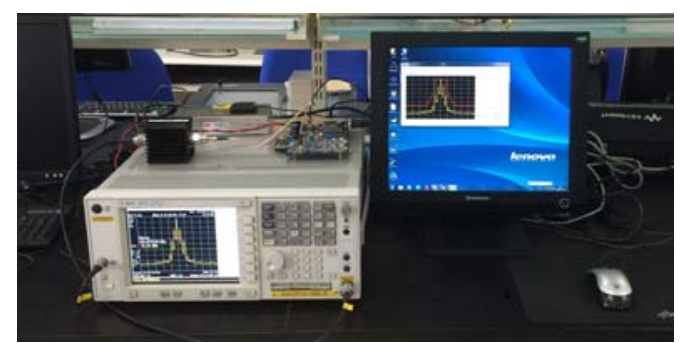

FIGURE II. ARCHITECTURE OF THE AIS PERFORMANCE AUTOMATIC TEST SYSTEM.

\section{SOFTWARE DESIGN AND IMPLEMENTATION}

Once the system architecture has been built, the next step is to pay attention to the software design and implementation that brings the whole test system to life.

\section{A. The Key Conditions of System}

In order to realize the communication between the scientific instrument and the computer, the software needs to fulfill two conditions. The first one is an installation of VISA on the computer. The second condition is having the SCPI used.

1) VISA: VISA is a library of functions and protocols which allows a successful communication between the computer and an instrument, and therefore it is an indispensable component. As a general purpose I/O standard, VISA is independent of instruments and suitable for various hardware interfaces such as LAN, GPIB, RS-232 and other interfaces. Instrument operation is managed and implemented by a connection of the practical application and instrumental function through the VISA resource management. The software programming can accomplish diverse applications by calling the appropriate VISA functions, such as opening or closing a session with the instrument, writing commands to or reading data from the instruments.

2) SCPI: SCPI is a standard programming language based on the existing IEEE488.1 and IEEE 488.2 specifications, and it is the standardization of a variety of standard instrument programming languages. At present, domestic and foreign researchers and producers of instruments commonly follow the standard. SCPI is transparent for instruments and hardware interfaces, and its function in cooperation with VISA is complementary to each other, thereby achieving remote control spectrum analyzer included in the test platform.

\section{B. Software Flow Chart}

The core programming principle of this software is using the VISA function library to deliver SCPI commands to the spectrum analyzer through the LAN interface. Then the instrument can make the corresponding response after receiving orders. Finally, measurement data will be returned to the computer through the LAN interface. The application is developed in Qt Creator, so exported data can be immediately available for further processing. Figure 3 is the software flow chart of the AIS AtoN Station Test Platform. 


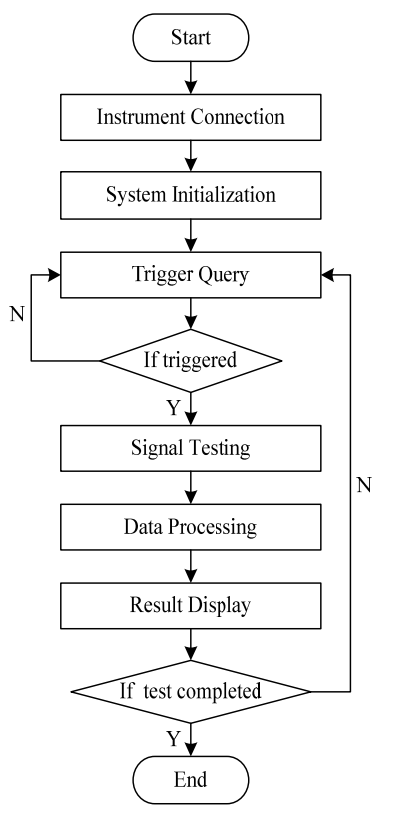

FIGURE III. SOFTWARE FLOW CHART OF THE AIS ATON STATION TEST PLATFORM.

In operation, at the first step, the spectrum analyzer will be connected to a computer via LAN. The corresponding IP address of the spectrum analyzer will be extracted and integrated into the format of a VISA resource name. Then the function of VISA Open is used to open a session with the instrument which the VISA resource name represents. The connection state can be shown both by the indicator light and status bar on the interface of software. After successful connection, instrument and software interface can be initialized. SCPI commands are sent to the spectrum analyzer to reset the instrument, configure appropriate value of parameters, and set the measurement mode as required.

Since the spectrum analyzer has already selected the trigger source and specified the trigger level via the remote control commands in the previous step, a trigger query is necessary to make sure of the real-time measurement before the real start of the signal testing. The trigger query command sets bit ' 0 ' in the standard event status register to ' 1 ' when the trigger has arrived. New commands are stopped from being processed until the pending operation is finished.

When spectrum analyzer has been triggered, the measurement commands according to the test requirement will be immediately sent to spectrum analyzer by the function of VISA Write, and the value of relevant parameters will be orderly got back by the function of VISA Read. The measurement data from spectrum analyzer are classified and saved in files automatically. Data storage is convenient for users to refer back to at a later time. Data processing is mainly used to determine the test results by separating, calculating, comparing, judging and other analysis methods based on the data returned, and for this reason, it plays a very important role to improve the test efficiency and precision.

A sufficient number of sweeps shall be used and sufficient transmission packets shall be measured to ensure that the emission profile is developed, and thus the test need sufficient data by measuring multiple times and obtaining a processed value. These measurement data returned and the spectrum waveform can be dynamically displayed on the software interface, and the final test result will not be calculated and displayed until enough tests of specified times have been executed. If the test has not finished, the device will wait for the next trigger.

\section{Test AND EVAluation OF THE System}

\section{A. Testing Results}

Based on the system architecture and software test platform which have described in above sections, several tests of AIS transmitter performance parameters, namely frequency error, carrier power and modulation spectrum slotted transmission, can be executed by this AIS performance automatic test system. Users can select the testing item optionally by switching the tab widget on the software interface.

TABLE I. SUMMARY OF PARAMETER SETTINGS

\begin{tabular}{|c|c|c|c|}
\hline \multirow[b]{2}{*}{ Parameter Settings } & \multicolumn{3}{|c|}{ Testing Items } \\
\hline & $\begin{array}{l}\text { Frequency } \\
\quad \text { error }\end{array}$ & $\begin{array}{l}\text { Carrier } \\
\text { Power }\end{array}$ & $\begin{array}{l}\text { Modulation } \\
\text { spectrum } \\
\text { transmission }\end{array}$ \\
\hline Center Frequency (MHz) & $\begin{array}{c}161.975 / \\
162.025 \\
\end{array}$ & $\begin{array}{c}161.975 / \\
162.025 \\
\end{array}$ & $\begin{array}{c}161.975 / \\
162.025 \\
\end{array}$ \\
\hline Span (kHz) & 5 & 1000 & 125 \\
\hline $\begin{array}{l}\text { External Amplifier Gain } \\
\text { (dB) }\end{array}$ & -30 & -30 & -30 \\
\hline Reference Level (dBm) & 50 & 50 & 50 \\
\hline $\begin{array}{l}\text { Resolution Bandwidth } \\
(\mathrm{kHz})\end{array}$ & auto-coupled & auto-coupled & 1 \\
\hline Video Bandwidth (kHz) & auto-coupled & auto-coupled & 3 \\
\hline Number of Sweep Points & $\begin{array}{l}\text { default } \\
\text { preset }\end{array}$ & $\begin{array}{l}\text { default } \\
\text { preset }\end{array}$ & 601 \\
\hline Trigger Mode & video & video & video \\
\hline Trigger Level (dBm) & -20 & -35 & -10 \\
\hline Trace Mode & $\begin{array}{l}\text { default } \\
\text { preset }\end{array}$ & $\begin{array}{l}\text { default } \\
\text { preset }\end{array}$ & max hold \\
\hline Sweep Type & auto-coupled & auto-coupled & $\begin{array}{l}\text { best speed / } \\
\text { fast Fourier } \\
\text { transform } \\
(\mathrm{FFT})\end{array}$ \\
\hline Default Test Times & 5 & 5 & 10 \\
\hline Marker & peak search & peak search & trace data \\
\hline Data to Read & $\begin{array}{c}\text { value of X- } \\
\text { axis }(\mathrm{Hz})\end{array}$ & $\begin{array}{l}\text { value of Y- } \\
\text { axis (dBm) }\end{array}$ & $\begin{array}{c}\text { Y-axis } \\
\text { amplitude } \\
\text { list }(\mathrm{dBm})\end{array}$ \\
\hline
\end{tabular}

During the testing process, the configuration parameters and measurement mode of the instrument and other initial conditions should be set according to the international standard IEC 62320-2 and practical testing experience. The summary of parameter settings are tabulated in Table 1. Each testing items has thirteen parameter settings such as center frequency, span, etc. Among them, the parameter value of the external amplifier gain depends on the attenuation value of the attenuator 
connected to the spectrum analyzer. The last line in Table 1 gives the data to read on the instrument display screen. Data on different axes of coordinate represent different measured values, for example, value of $\mathrm{X}$-axis represents the frequency and value of $\mathrm{Y}$-axis represents the corresponding amplitude.

The software interface are shown in Figure 4. The final test results can be displayed on the software interface and automatically stored in files. Testers can get the AIS performance parameters accurately, clearly and directly. In Figure 4, part (a) shows the value and test result of frequency error, part (b) shows the value and test result of carrier power, and part (c) shows the spectrogram and test result of modulation spectrum slotted transmission.

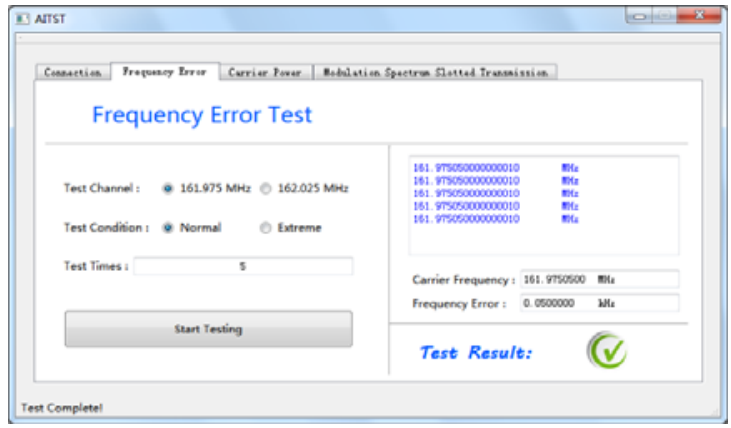

(a)

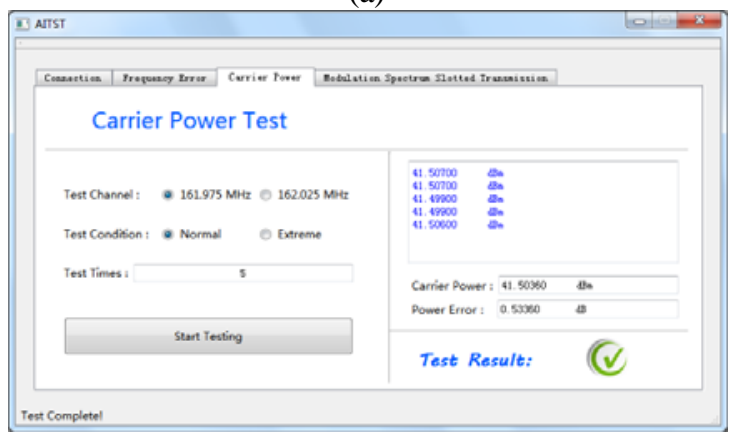

(b)

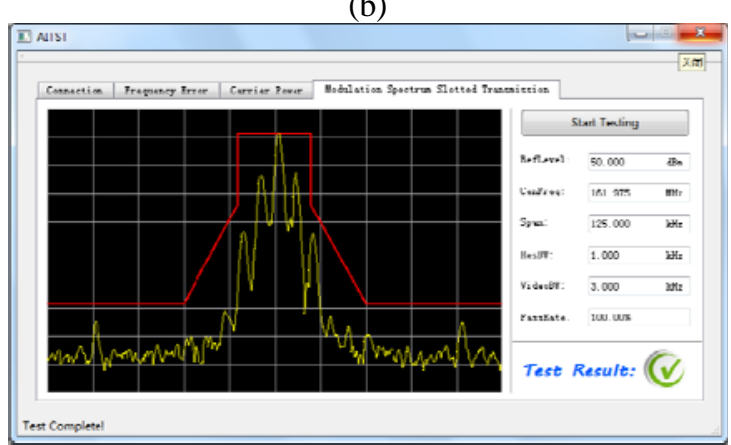

(c)

FIGURE IV. THE FINAL TESTING RESULTS ON THE SOFTWARE INTERFACE.

\section{B. Performance Evaluations}

Compared with the traditional AIS performance testing method, the remote automatic test System with high precision for AIS performance in this paper makes the test efficiency and accuracy of measurement improved significantly.
1) Efficiency of testing: In order to conquer the difficulties of manual testing, and enhance the efficiency of AIS performance testing, the remote control and automation of testing process is applied in this test system .

a) Remote control testing: Manual testing method requires experienced testers have to be physically present in the testing place and do a field test. In this study, the application of LAN connects the computer, terminals and other required equipment into a net within the limited scope (for example, a laboratory, a building or a campus). And then, the measuring instrument can be configured, monitored and managed by a remote control software developed in this study Remote control testing can break through the limitation of traditional testing resources and testing method. Without the restraint of time and space, researchers may experience the efficient testing, and enjoy the great convenience.

b) Automation software testing: By manual testing method, all of the parameters on the spectrum analyzer need to be manually configured one by one before starting each testing of AIS performance. To guarantee the measurement accuracy, it is necessary to repeat the measurement and do some data processing, which means the tester have to manually deal with all work during the test, such as recording each measured value, calculating the mean value and the error value, getting the final result by comparing with the specified value on the testing standard. It is clear that the entire testing process is quite complicated and time-consuming. Only one testing item, such as AIS transmitter frequency error, could take at least ten minutes, even if we start the time when testers have already arrived at the testing place and connected all the instruments and equipment. However, the testing efficiency of AIS performance has a qualitative leap owing to the introduction of "automation software testing". Ten-minute operation procedure including data recording, calculation and comparison can be completed automatically and compressed into a few seconds by using a computer with the designed ATE control software. As a result, the testing efficiency has been dramatically enhanced.

2) Precision of measurement: In order to improve the measurement accuracy of AIS performance, this study has been improved in the following aspects:

a) Real-time measurement: The existing automatic testing platform mostly adopted the way of taking regular readings by a timer to collect data, and by this approach, continuous testing can be realized. However, all of the measurement data will be lost in case of a test signal emission to the measuring instruments during the interval between two read operations. A new method of data acquisition is designed in this study to prevent data loss in the process of measurement, and we named it "trigger query". Through this method, the testing software can determine whether there is a test signal by querying whether the measuring instruments is triggered. Hence, the problem of data missing is solved effectively, and to some extent, the precision of measurement is enhanced by the guarantee of real-time. 
b) Quantitative measurement: The quantitative measurement presented in this study brings a considerable improvement of the transmission spectrum measurement precision. The bandwidth allocated for AIS transmissions is defined by a "spectrum mask". The mask is a set of boundary lines marked on a frequency domain plot of the AIS transmitter output power. To meet the international standard IEC 62320-2, the accumulated transmitter spectral emission captured on the spectrum analyzer should not be higher than the mask. In the existing measuring method, this test result was determined mainly by naked eyes without convincing data. However, this AIS performance automatic test system is able to set arbitrary number of data points as well as compare the trace data of the transmission spectrum point by point.

The number of sweep points is set to 600 in this design, and due to the large number of data points are difficult to list, only seven sweep points of each measured value group are taken as sample points and tabulated in Table 2. These points are selected at the inflection points, the center point and the boundary points of the mask. The first row in Table 2 is the relative value of required power result at its corresponding frequency according to the AIS test standard, and its unit is $\mathrm{dBc}$. The second row in Table 2 is the absolute value of required power result after conversion according to the reference level, and its unit is $\mathrm{dBm}$ typically.

TABLE II. SUMMARY OF MEASURED DATA ON TRANSMISSION SPECTRUM MEASUREMENT

\begin{tabular}{|c|c|c|c|c|c|c|c|}
\hline $\begin{array}{c}\text { Require } \\
\text { d Result } \\
\text { (dBc) }\end{array}$ & -60 & -60 & -25 & 0 & -25 & -60 & -60 \\
\hline $\begin{array}{c}\text { Require } \\
\text { d Value } \\
\text { (dBm) }\end{array}$ & -19.03 & -19.03 & 15.97 & 42.47 & 15.97 & -19.03 & -19.03 \\
\hline \multirow{10}{*}{$\begin{array}{c}\text { Measur } \\
\text { ed } \\
\text { Value } \\
(\mathrm{dBm})\end{array}$} & -38.587 & -32.291 & -8.395 & 33.885 & 4.662 & -29.803 & -37.997 \\
\hline & -37.85 & -32.291 & -8.395 & 33.885 & 4.662 & -29.803 & -37.997 \\
\hline & -42.39 & -34.884 & -8.413 & 33.907 & 4.74 & -31.759 & -37.015 \\
\hline & -39.471 & -32.879 & -8.581 & 33.839 & 4.755 & -30.643 & -40.57 \\
\hline & -42.797 & -34.398 & -8.712 & 33.912 & 4.763 & -30.166 & -38.97 \\
\hline & -39.321 & -34.757 & -8.428 & 33.877 & 4.831 & -28.417 & -41.539 \\
\hline & -39.454 & -32.423 & -8.735 & 33.869 & 4.84 & -30.585 & -30.804 \\
\hline & -39.474 & -35.523 & -8.834 & 33.834 & 4.805 & -31.101 & -39.379 \\
\hline & $39 . \overline{4} 18$ & -32.065 & -8.326 & 33.895 & 4.776 & -29.243 & -37.855 \\
\hline & -37.85 & -32.291 & -8.395 & 33.885 & 4.729 & -29.173 & -37.997 \\
\hline
\end{tabular}

The test result will not be qualified unless all the test points on trace must be entirely within the mask, which means the pass rate must be 100 percent. Due to the quantitative calculation instead of the qualitative analysis, the system realized the high precision test of the modulation spectrum slotted transmission, specifically when the transmission spectrum is very close to the limit line and the spacing error is invisible. The part (c) in Figure 4 gives a clear indication of the testing result with a definite pass rate.

\section{CONCLUSIONS}

The AIS performance automatic test system presented in this paper has realized the remote control of the spectrum analyzer by using the SCPI commands and VISA programming technology through the LAN interface. The software platform established can complete the automatic test of AIS transmitter performance, which is time-saving and costeffective. And meanwhile, it also provides a friendly interface for users to operate more expediently and get the results more intuitively. The design of the test system features real-time, high precision, easy expansibility, etc. Moreover, the test system is developed on the basis of international standard, and it can be more fully functional in depth further. Thus, this automatic test system has practical value and a good prospect in the field of AIS performance test.

\section{ACKNOWLEDGMENT}

This research was supported by the key project National Science Foundation of China, No.6123006 and No.801104.

\section{REFERENCES}

[1] Qing, Hu; Jiang, Yi; Zhang, Jingbo, "Development of an Automatic Identification System Autonomous Positioning System,” in SENRSOS, vol.15, issue:11 pp.28574-28591, NOV 2015.

[2] Class B AIS - Critical performance tests, SRT Marine Technology Ltd, 2011.

[3] Wei Fu; Jun Hu; Shuhan Zhang, "Frequency-domain measurement of 60 $\mathrm{GHz}$ indoor channels: a measurement setup, literature data, and analysis,” in Instrumentation \& Measurement Magazine, IEEE, vol.16, no.2, pp.34-40, April 2013.

[4] Shejbal, T.; Petkov, M.; Zalabsky, T.; Pidanic, J.; Zaplatilek, L., "The workplace for automatic measurement of antennas' radiation patterns,” in Radioelektronika (RADIOELEKTRONIKA), 2014 24th International Conference, pp.1-4, 15-16 April 2014.

[5] Kang Jianhua; She Fangyi; Zhang Weidong; Ping Jie, “Application of Network Analyzer Based on the Automatic Control of LabVIEW in Radio and Television Test," in Biometrics and Security Technologies (ISBAST), 2013 International Symposium on, pp.14-19, 2-5 July 2013.

[6] Liu Guili; Kong Quancun, "Design of virtual oscilloscope based on GPIB interface and SCPI," in Electronic Measurement \& Instruments (ICEMI), 2013 IEEE 11th International Conference on , vol.1, pp.294298, 16-19 Aug. 2013.

[7] Jing, Huang; Leling, Qiao; Hanbo, Zhang, "Research of Automatic Digital Multimeter Interface Technology Based on UART," in Networking and Distributed Computing (ICNDC), 2012 Third International Conference on, pp.108-110, 21-24 Oct. 2012.

[8] Xiufang Wang; Lin Dai, "Design of wireless signal analysis system based on intelligent control,” in Measurement, Information and Control (ICMIC), 2013 International Conference on, vol.02, pp.1180-1183, 1618 Aug. 2013.

[9] IEC 62320-2 INTERNATIONAL STANDARD, maritime navigation and radiocommunication equipment and systems, Part 2: AIS AtoN Stations - operational and performance requirements, methods of testing and required test results, Geneva, Switzerland, 2008. Available online: http://www.iec.ch 